Please quote as: Gebauer, L.; Söllner, M. \& Leimeister, J. M. (2016): Vertrauensproblematiken im Cloud-Computing-Umfeld. In: Cloud-Services aus der Geschäftsperspektive. Hrsg./Editors: Krcmar, H.; Leimeister, J. M.; Roßnagel, A. \& Sunyaev, A. Verlag/Publisher: Springer Gabler, Wiesbaden. Erscheinungsjahr/Year: 2016. Seiten/Pages: 59-69. 


\title{
6 Vertrauensproblematiken im Cloud-Computing-Umfeld
}

\author{
L. Gebauer, M. Söllner, J.M. Leimeister
}

\subsection{Die Bedeutung von Vertrauen im Cloud-Computing-Umfeld}

Vier Jahre in Folge war Cloud-Computing, gemäß der jährlich durchgeführten BITKOM-Umfrage „IT Trends des Jahres“, das wichtigste Thema in der IKT-Branche (BITKOM 2010, 2011, 2012, 2013). Cloud-Computing wird gehandelt als „long-held dream of computing as a utility“ (Armbrust et al. 2010, S. 50), der sich endlich bewahrheitet. Es bietet Unternehmen eine Lösung, um hohe Wartungskosten zu senken, die Auslastung und Nutzung der Hardwareressourcen zu optimieren, den internen Energieverbrauch zu verringern sowie agiler auf geänderte Geschäftsanforderungen $\mathrm{zu}$ reagieren (IDC 2011). Dennoch zeigen Studien und Umfragen, dass Cloud-Computing im B2B-Bereich eher zurückhaltend genutzt wird (Golkowsky/Vehlow 2011; IDC 2011). Vor allem der Mittelstand hat noch enormen Aufholbedarf (BITKOM 2014).

Die bisherige Forschung zeigt, dass eine Zurückhaltung bei der Akzeptanz und Nutzung neuer Technologien sowohl auf einen Mangel an Vertrauen (z.B. Söllner/Leimeister 2012; Gefen et al. 2003) als auch auf die Wahrnehmung von Risiken (z.B. Featherman/Pavlou 2003; Luo et al. 2010) zurückzuführen ist. Vertrauen und Risiko sind zwei voneinander unabhängige Wahrnehmungsgrößen (Joubert/Belle 2009), die sich dennoch gegenseitig beeinflussen. Das Maß an entgegengebrachtem Vertrauen wirkt sich positiv auf die Bereitschaft Risiken einzugehen aus (z.B. Glover/Benbasat 2010).

Auch im Zuge der Akzeptanz und Nutzung von Cloud-Computing bestehen Risiken und Hemmnisse seitens der (potenziellen) Kunden (z.B. Gebauer et al. 2012; Armbrust et al. 2010; Ackermann et al. 2011), wobei die Einschätzung der Vertrauenswürdigkeit als ein erfolgskritischer Faktor bei der Auswahl des Cloud-Computing-Anbieters gesehen wird (Repschlaeger et al. 2012). Nach dem Trendforscher Sven Gábor Jánszky ist Cloud-Computing ein Geschäft des Vertrauens, denn ,die Frage, welchem Unternehmen der 
Kunde erlaubt, seine Daten zu verwalten, seine Recommendation-Systeme anzubieten und seine elektronischen Assistenten zu installieren, wird zur strategisch wichtigsten Marktmacht" (Jánszky 2012, S. 4). Dieser Kommentar bestärkt, dass Vertrauen bzw. Vertrauenswürdigkeit zu einem der wichtigsten Erfolgsfaktoren von Cloud-Computing-Anbietern avancieren wird.

Um sowohl die Akzeptanz als auch die Nutzung von Cloud-Computing im B2B-Bereich, speziell im deutschen Mittelstand, zu erhöhen, sollten dementsprechend die Anbieter von Cloud-Computing-Diensten die Nutzungshemmnisse der (potenziellen) Kunden bestmöglich adressieren und gleichzeitig Maßnahmen zur Vertrauensförderung einsetzen. Um die Cloud-Computing-Anbieter bei dieser Aufgabe zu unterstützen, werden in den nachfolgenden Abschnitten die Nutzungshemmnisse für Cloud-Computing zusammenfassend erläutert. Anhand der verschiedenen Komponenten von Vertrauen (siehe Abschnitt 2.3.3) wird abgeleitet, zu welchen Entitäten im CloudComputing-Umfeld die (potenziellen) Kunden Vertrauen aufbauen müssen. Abschließend werden die herausgestellten Nutzungshemmnisse für CloudComputing im B2B-Bereich den identifizierten Vertrauensbeziehungen zugeordnet.

\subsection{Nutzungshemmnisse im Cloud-Computing-Umfeld}

Um einen umfassenden Überblick über die Nutzungshemmnisse gegenüber Cloud-Computing im B2B-Bereich zu geben, wurden die Erkenntnisse von drei verschiedenen Überblicksartikeln (Armbrust et al. 2010; Ackermann et al. 2011; Marston et al. 2011) zusammengefasst. Die Überblicksartikel betrachten die Nutzungshemmnisse aus verschiedenen Perspektiven. Armbrust et al. (2010) zeigen generelle Hindernisse bei der Nutzung von CloudComputing auf, Ackermann et al. (2011) betrachten die Risiken aus technischer Perspektive und Marston et al. (2011) aus betriebswirtschaftlicher Sicht.

Die anhand der Überblicksartikel gewonnenen Erkenntnisse wurden miteinander auf Überschneidungen und Unterschiede verglichen, kategorisiert und in einer Tabelle zusammengefasst (Tabelle 1). Im Ergebnis wurden zwölf 
Tabelle 1: Hemmnisse für die Nutzung von Cloud-Computing im B2B-Bereich (Quelle: Gebauer et al. 2012)

\begin{tabular}{|c|c|c|}
\hline Hemmnis & Beschreibung & Quelle \\
\hline $\begin{array}{l}\text { Diskonti- } \\
\text { nuität/ } \\
\text { Ausfall des } \\
\text { Dienstes }\end{array}$ & $\begin{array}{l}\text { Störungen des Betriebsalltags sowie von betriebs- } \\
\text { notwendigen Anwendungen durch temporäre Unter- } \\
\text { brechungen oder komplette Ausfälle des Dienstes }\end{array}$ & $A, B, C$ \\
\hline $\begin{array}{l}\text { System- } \\
\text { angriffe }\end{array}$ & $\begin{array}{l}\text { Gefährdung der Informationssicherheit durch ex- } \\
\text { terne Systemangriffe, Anbieter verantwortlich für } \\
\text { Bereitstellung und Funktionsfähigkeit geeigneter } \\
\text { Sicherheitstechniken }\end{array}$ & $A$ \\
\hline $\begin{array}{l}\text { Netzwerk- } \\
\text { störungen }\end{array}$ & $\begin{array}{l}\text { Störungen durch nicht ausreichende Bereitstellung } \\
\text { von Netzwerkbandbreite (z.B. Schwankungen, Aus- } \\
\text { fälle, zu lange Ladezeiten bei der Bearbeitung von } \\
\text { Prozessen) }\end{array}$ & $A, B$ \\
\hline $\begin{array}{l}\text { Begrenzte } \\
\text { Skalier- } \\
\text { barkeit }\end{array}$ & $\begin{array}{l}\text { Begrenzte oder zu langsame Adaption des Dienstes } \\
\text { an aktuelle Leistungsanforderungen vom Kunden } \\
\text { (z.B. flexibler Bedarf an Speicherplatz und Rechen- } \\
\text { leistung) }\end{array}$ & $A, B$ \\
\hline $\begin{array}{l}\text { Daten- } \\
\text { übertragung }\end{array}$ & $\begin{array}{l}\text { Zeit- und kostenintensive Übertragung großer } \\
\text { Datenmengen (z.B. im Terabyte-Bereich) über das } \\
\text { Netzwerk }\end{array}$ & $B$ \\
\hline $\begin{array}{l}\text { Mangelnde } \\
\text { Daten- } \\
\text { integrität }\end{array}$ & $\begin{array}{l}\text { Mangelnde Transparenz hinsichtlich der Datenüber- } \\
\text { tragung, Datenmodifikation und Zugriffshierarchien. } \\
\text { Anbieter verantwortlich für korrekte Identifizierung, } \\
\text { Authentifizierung und Autorisierung von Nutzern zur } \\
\text { Verhinderung von Datenmissbrauch und unautori- } \\
\text { sierten Zugriffen }\end{array}$ & $A, B$ \\
\hline $\begin{array}{l}\text { Abhängig- } \\
\text { keit vom } \\
\text { Anbieter }\end{array}$ & $\begin{array}{l}\text { Datenverteilung auf mehrere Cloud-Computing-An- } \\
\text { bieter nicht möglich, starke Anwenderabhängigkeit } \\
\text { vom Anbieter, Gefahren für Anwender: willkürliche } \\
\text { Preisfestsetzung, Reliabilitätsprobleme, Anbieter- } \\
\text { bankrott, etc. }\end{array}$ & $B$ \\
\hline $\begin{array}{l}\text { Abhängig- } \\
\text { keit von } \\
\text { anderen } \\
\text { Nutzern }\end{array}$ & $\begin{array}{l}\text { Viele Kunden nutzen die gleiche Cloud, wobei Fehl- } \\
\text { verhalten von Nutzern auch Auswirkungen für die } \\
\text { restlichen Nutzer haben kann, z.B. verstößt ein } \\
\text { Cloud-Computing-Nutzer gegen geltendes Recht, } \\
\text { sind staatliche Behörden befugt den gesamten } \\
\text { Server zu beschlagnahmen }\end{array}$ & $B$ \\
\hline
\end{tabular}




\begin{tabular}{|l|l|l|}
\hline Hemmnis & Beschreibung & Quelle \\
\hline $\begin{array}{l}\text { Kontroll- } \\
\text { möglich- } \\
\text { keiten }\end{array}$ & $\begin{array}{l}\text { Mangelnde Kontrolle über Speicherort, Updates und } \\
\text { sonstige automatische Wartungsarbeiten }\end{array}$ & A, B, C \\
\hline $\begin{array}{l}\text { Mangelnde } \\
\text { Reliabilität }\end{array}$ & $\begin{array}{l}\text { Mangelndes Vertrauen, ob das System gemäß den } \\
\text { Nutzererwartungen operiert (z.B. ungenügende } \\
\text { Softwarequalität, unzureichende Leistung) }\end{array}$ & A, C \\
\hline $\begin{array}{l}\text { Mangelnde } \\
\text { rechtliche } \\
\text { Standards }\end{array}$ & $\begin{array}{l}\text { Keine einheitlichen Standards, Gesetze und Ver- } \\
\text { träge hinsichtlich Datenschutz, Datensicherheit, } \\
\text { Datenzugriff, Datenlokation, Anbieterwechsel, } \\
\text { Anbieterbankrott, etc. }\end{array}$ & A, B, C \\
\hline $\begin{array}{l}\text { Gefährdung } \\
\text { interne IT }\end{array}$ & $\begin{array}{l}\text { Gefährdung der internen IT in Bezug auf Daten- } \\
\text { sicherheit, Kompatibilität und Jobsicherheit }\end{array}$ & C \\
\hline $\begin{array}{l}\text { Anmerkung: Quelle A entspricht Ackermann et al. (2011), Quelle B entspricht } \\
\text { Armbrust et al. (2010) und Quelle C entspricht Marston et al. (2011). }\end{array}$ \\
\hline
\end{tabular}

Kategorien an Nutzungshemmnissen gegenüber Cloud-Computing im B2BBereich identifiziert (Gebauer et al. 2012). In der ersten Spalte der Tabelle wurden die Nutzungshemmnisse stichwortartig zusammengefasst und in der zweiten Spalte detailliert beschrieben. In der dritten Spalte findet sich die Zuordnung, in welchen Quellen weitere Informationen zu den entsprechenden Hemmnissen zu finden sind.

\subsection{Vertrauensbeziehungen im Cloud-Computing-Umfeld}

Vertrauen setzt sich aus drei verschiedenen Komponenten zusammen (vgl. Leimeister et al. 2005): Vertrauensbereitschaft, interpersonelles Vertrauen und Systemvertrauen (siehe Kapitel 2). Vertrauen in komplexe technische Systeme wird als Netzwerk verschiedener Vertrauensbeziehungen verstanden (Muir 1994), da an der Leistungserbringung verschiedenste Entitäten beteiligt sind und gegenseitige Vertrauensbeziehungen etabliert werden müssen (z.B. Söllner/Leimeister 2012). Als Entitäten werden in der Informatik Objekte bezeichnet, die eindeutig identifiziert werden können, wie eine Person, ein Unternehmen, ein Ereignis, ein Zustand, eine Sache oder Gegenstand (Chen 1976). Ziel ist, anhand der verschiedenen Komponenten von Vertrau- 
en, ein Vertrauensnetzwerk für das Cloud-Computing-Umfeld zu entwickeln, um herauszustellen, zu welchen Entitäten ein (potenzieller) Cloud-Computing-Kunde Vertrauen aufbauen muss.

Die Komponente Vertrauensbereitschaft umfasst die grundsätzliche Bereitschaft Anderen zu vertrauen. Diese entwickelt sich sowohl durch Erfahrungen im alltäglichen Leben als auch durch Persönlichkeitsmerkmale und wird als eine relativ stabile Persönlichkeitseigenschaft betrachtet (Rotter 1980). Die Vertrauensbereitschaft hat einen Einfluss auf die Ausprägung von Vertrauen (z.B. Kim et al. 2008; Joubert/Belle 2009) und sogar auf die Nutzungsabsicht (z.B. Luo et al. 2010). Da diese Vertrauenskomponente als stabile Persönlichkeitseigenschaft anzusehen ist, kann diese durch Maßnahmen des Cloud-Computing-Anbieters nicht adressiert werden und wird deshalb im Weiteren nicht tiefgehender betrachtet.

Die Vertrauenskomponenten interpersonelles Vertrauen und Systemvertrauen hingegen sind durch den Anbieter gestaltbar (Joubert/Belle 2009; Söllner/Leimeister 2012; Leimeister et al. 2005). Diese machen deutlich, dass der (potenzielle) Cloud-Computing-Nutzer eine Vertrauensbeziehung zum Cloud-Computing-Anbieter als Person (interpersonelles Vertrauen) und eine Vertrauensbeziehung zur Technik selbst (Systemvertrauen) aufbauen muss (vgl. Gebauer et al. 2012). Daraus ergeben sich die ersten beiden Entitäten des Vertrauensnetzwerks im Cloud-Computing-Umfeld (Abbildung 1).

Im Cloud-Computing-Umfeld bestehen noch zwei weitere Besonderheiten, die es bei der Entwicklung des Vertrauensnetzwerks zu berücksichtigen gilt. Zum einen können die (potenziellen) Cloud-Computing-Nutzer den Dienst nur zuverlässig nutzen, wenn eine ausreichende und dauerhafte Internetverbindung sichergestellt ist. Dementsprechend ist die dritte Entität, zu der Cloud-Computing-Nutzer eine Vertrauensbeziehung aufbauen müssen, der ausgewählte Netzbetreiber. Unter Netzbetreibern werden die verschiedenen Internetanbieter, die am Markt existieren, zusammengefasst. Cloud-Computing-Dienste werden über das Internet bereitgestellt, weshalb den Netzbetreibern eine erfolgskritische Rolle zukommt. Gelingt es dem Netzbetreiber des Cloud-Computing-Nutzers nicht, kontinuierlich die geforderte Bandbreite zur Verfügung zu stellen, kann dies zu Diskontinuitäten bis hin zu 


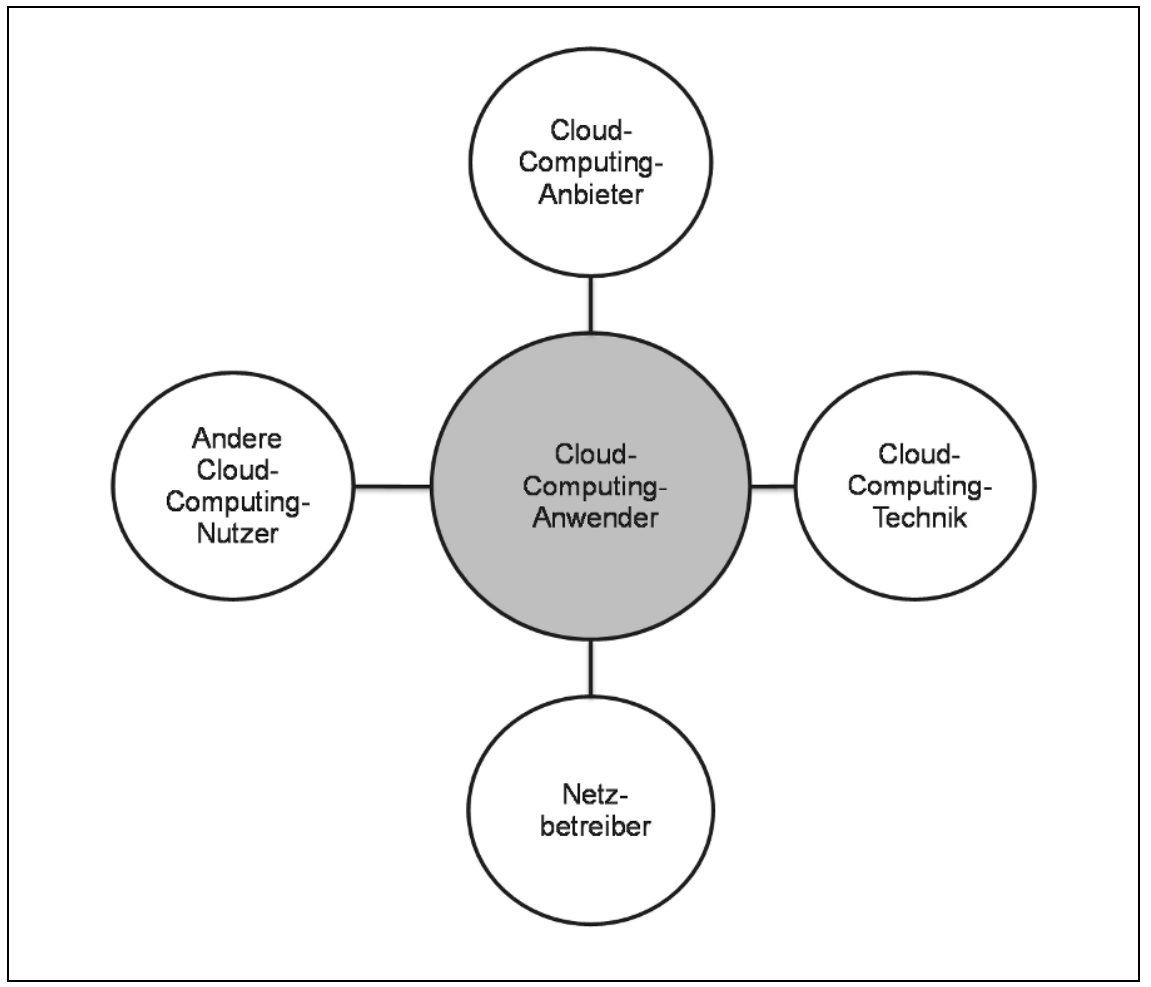

Abbildung 1: Vertrauensbeziehungen im Cloud-Computing-Umfeld (Quelle: Gebauer et al. 2012)

kompletten Ausfällen führen. Dadurch ist die Sicherheit der Betriebsabläufe durch die Nutzung von Cloud-Computing gefährdet. Die Vertrauensbeziehung des Cloud-Computing-Kunden zu seinem Netzbetreiber bezieht sich darauf, dass dieser stets eine ausreichende Bandbreite zur Verfügung stellt, um Störungen des Betriebsalltages auszuschließen.

Die zweite Besonderheit im Cloud-Computing-Umfeld besteht darin, dass verschiedene Nutzer auf ein und die gleiche Cloud zugreifen können. Demzufolge besteht bei Fehlverhalten oder rechtswidrigen Handlungen von anderen Cloud-Computing-Nutzern die Gefahr, dass die Nutzung des CloudComputing-Dienstes unvermittelt durch die Behörden für alle Nutzer gesperrt werden kann (siehe Kapitel 18). Dies hat zur Folge, dass (potenzielle) 
Cloud-Computing-Nutzer ebenfalls eine Vertrauensbeziehung $\mathrm{zu}$ den anderen Cloud-Computing-Nutzern aufbauen müssen. Dementsprechend muss sich die Vertrauensbeziehung des Cloud-Computing-Nutzers zu den anderen Nutzern darauf beziehen, dass diese ebenfalls hinreichend Verantwortung für einen reibungslosen Zugriff tragen.

Zusammenfassend müssen (potenzielle) Cloud-Computing-Nutzer vier Vertrauensbeziehungen aufbauen (Gebauer et al. 2012): zum Cloud-Computing-Anbieter, zum technischen System, zum ausgewählten Netzbetreiber und schließlich auch zu den anderen Nutzern der Cloud (Abbildung 1).

\subsection{Zuordnung der Nutzungshemmnisse zu den Vertrauensbeziehungen}

In diesem Abschnitt werden nun die identifizierten Nutzungshemmnisse den Entitäten des Vertrauensnetzwerks zugeordnet (Abbildung 2). Die überwiegende Anzahl der Nutzungshemmnisse können direkt durch den CloudComputing-Anbieter adressiert werden. Dieser kann mit Hilfe geeigneter Maßnahmen versuchen, die Hemmnisse der (potenziellen) Cloud-Computing-Anwender abzubauen. Zum Beispiel, indem dieser detailliert darlegt, welche Schutzmaßnahmen vor Systemangriffen durchgeführt werden, die Anwender rechtzeitig über Updates und dadurch bedingte Betriebsstörungen informiert oder die Nutzer über die rechtlichen Standards aufklärt.

Der Cloud-Computing-Anbieter ist ebenfalls dafür verantwortlich, die Funktionsfähigkeit der von ihm angebotenen Cloud-Computing-Technik sicherzustellen. Dennoch wird die Cloud-Computing-Technik als eigene Entität betrachtet, weil der (potenzielle) Cloud-Computing-Kunde unabhängig vom Anbieter auch der Cloud-Computing-Technik an sich vertrauen muss. Gegenüber der Technik bestehen die Nutzungshemmnisse Diskontinuität/ Ausfall des Dienstes und mangelnde Reliabilität. Ein wesentliches Nutzungshemmnis gegenüber Cloud-Computing besteht darin, dass der Betriebsalltag enorm beeinträchtigt ist, wenn die Bereitstellung gestört ist oder gar ausfällt, da betriebsnotwendige und geschäftskritische Anwendungen nicht zur Verfügung stehen. Auch bestehen generelle Bedenken, ob die Cloud-Computing-Technik gemäß den Nutzererwartungen operiert. 


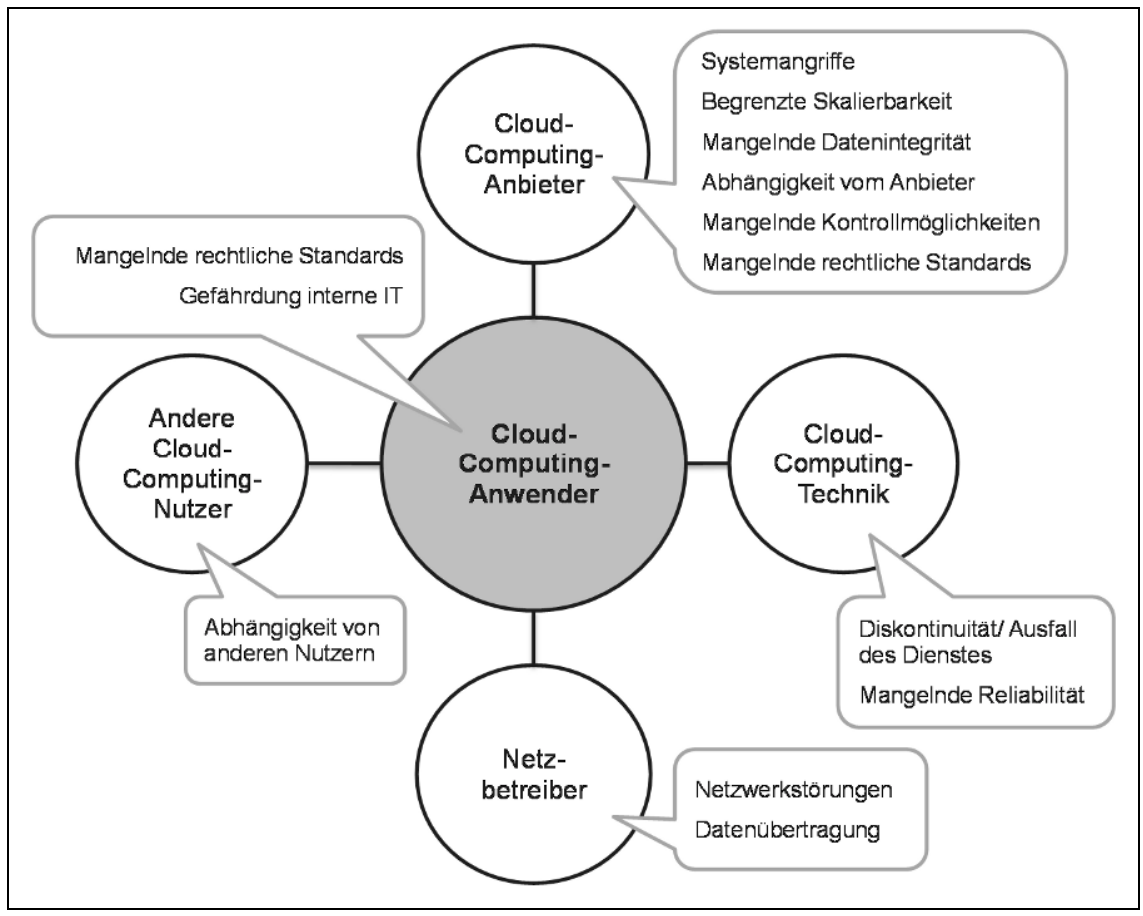

Abbildung 2: Vertrauensbeziehungen im Cloud-Computing-Umfeld und Zuordnung der Nutzungshemmnisse (Quelle: Gebauer et al. 2012)

Neben den Cloud-Computing-Anbietern wird das Nutzungshemmnis mangelnde rechtliche Standards zusätzlich den Cloud-Computing-Nutzern zugeordnet, da diese in der eigenen Verantwortung stehen, sich über die aktuelle Rechtslage und die eigenen rechtlichen Pflichten im Zuge der Nutzung von Cloud-Computing zu informieren (siehe Kapitel 15 bis 18). Dennoch kann der Cloud-Computing-Anbieter den (potenziellen) Kunden proaktiv bei der Schaffung der rechtlichen Informiertheit und Sicherstellung von rechtmäßigem Verhalten unterstützen. Zudem sollte der (potenzielle) CloudComputing-Anwender vor der Nutzung sicherstellen, dass die Implementierung von Cloud-Computing-Diensten mit der bestehenden IT-Infrastruktur kompatibel ist und sich diesbezüglich mit dem Cloud-Computing-Anbieter abstimmen. 
Wie schon erwähnt, bestehen ebenfalls gegenüber den anderen CloudComputing-Nutzern Nutzungshemmnisse, da sich die Anwender eine Cloud teilen. Die Konsequenzen des Fehlverhaltens anderer Cloud-Computing-Nutzer können Auswirkungen auf die gesamte Cloud-Computing-Nutzerschaft haben. Speichert ein Cloud-Computing-Nutzer beispielsweise rechtswidrige Inhalte, sind die staatlichen Behörden befugt, die Cloud-Computing-Server als Beweismittel zu beschlagnahmen (siehe Kapitel 18). Auch kann es fahrlässig oder vorsätzlich passieren, dass Cloud-Computing-Nutzer die Server mit Schadprogrammen infizieren, die ebenfalls Konsequenzen für die gesamte Nutzerschaft haben (siehe Kapitel 17).

Durch diese Zuordnung wird deutlich, mit welchen Nutzungshemmnissen sich (potenzielle) Cloud-Computing-Kunden auseinander setzen und welche Entität diese prinzipiell zu verantworten hat. Auch wenn der Cloud-Computing-Anbieter nicht alle Hemmnisse auslöst, sollte dieser dennoch versuchen, alle Hemmnisse der (potenziellen) Cloud-Computing-Kunden zu adressieren, um mit Hilfe von vertrauensunterstützenden Maßnahmen sowohl das Vertrauen als auch die Akzeptanz zu fördern.

\subsection{Zusammenfassung}

Mit dem Kapitel „Vertrauensproblematiken im Cloud-Computing-Umfeld“ wurde ein Überblick geschaffen, welche Besonderheiten sich in Bezug auf Vertrauen im Cloud-Computing-Umfeld ergeben. Der Aufbau von Vertrauen und der Abbau von Nutzungshemmnissen können als erfolgskritische Faktoren für die Akzeptanz und die Nutzung von Cloud-Computing angesehen werden.

Daher wurden in diesem Kapitel die Hemmnisse gegenüber der Nutzung von Cloud-Computing-Diensten zusammengetragen und kategorisiert. Insgesamt wurden zwölf verschiedene Kategorien von Nutzungshemmnissen erarbeitet. Zudem wurde aufgezeigt, dass der (potenzielle) Cloud-ComputingAnwender aufgrund der Besonderheiten im Cloud-Computing-Umfeld nicht nur zu dem Cloud-Computing-Anbieter, sondern zu drei weiteren Entitäten (Cloud-Computing-Technik, Netzbetreiber und anderen Cloud-ComputingNutzern) Vertrauen aufbauen muss. 
Abschließend wurden die identifizierten Kategorien an Nutzungshemmnissen den entsprechenden Entitäten des Vertrauensnetzwerks zugeordnet, um aufzuzeigen, welche Entität das Hemmnis auslöst bzw. zu verantworten hat. Auch wenn eine derartige Zuordnung der Nutzungshemmnisse vorgenommen wurde, liegt es beim Cloud-Computing-Anbieter, diesen entgegenzuwirken und das Vertrauen in den angebotenen Cloud-Computing-Dienst zu fördern.

\subsection{Literaturverzeichnis}

Ackermann, T.; Miede, A.; Buxmann, P.; Steinmetz, R. (2011): Taxonomy of Technological IT Outsourcing Risks: Support for Risk Identification and Quantification. Präsentiert auf der 19th European Conference on Information Systems (ECIS), Helsinki, Finnland.

Armbrust, M.; Fox, A.; Griffith, R.; Joseph, A.D.; Katz, R.; Konwinski, A.; Lee, G.; Patterson, D.; Rabkin, A.; Stoica, I.; Zaharia, M. (2010): A view of cloud computing. In: Communications of the ACM, Vol. 53 (2010) No. 4, S. 50-58.

BITKOM (2010): IT- und Telekommunikations-Trends 2010. http://www.bitkom. org/files/documents/BITKOM-Presseinfo_IT-Trends_2010_-_13_01_2010.pdf, zugegriffen am 28.07.2014.

BITKOM (2011): Cloud Computing ist erneut IT - Trend des Jahres. http://www. bitkom.org/files/documents/BITKOM-Presseinfo_IT-Trends_2011_-_18_01_ 2011.pdf, zugegriffen am 28.07.2014.

BITKOM (2012): Die Hightech-Trends des Jahres 2012 http://www.bitkom.org/ files/documents/BITKOM-Presseinfo_IT-Trends_des_Jahres_18_01_2012.pdf, zugegriffen am 28.07.2014.

BITKOM (2013): Die wichtigsten Hightech - Themen 2013 http://www.bitkom. org/files/documents/BITKOM_Presseinfo_Hightech-Trends_16_01_2013.pdf, zugegriffen am 28.07.2014.

BITKOM (2014): Nutzung von Cloud Computing in Unternehmen wächst. http:// www.bitkom.org/files/documents/Presseinfo_BITKOM_und_KPMG_zum_Cloud -Monitor_30_01_2014.pdf, zugegriffen am 28.07.2014.

Chen, P.P.-S. (1976): The entity-relationship model - toward a unified view of data. In: ACM Transactions on Database Systems (TODS), Vol. 1 (1976) No. 1, S. 936.

Featherman, M.S.; Pavlou, P.A. (2003): Predicting e-services adoption: a perceived risk facets perspective. In: International Journal of Human-Computer Studies, Vol. 59 (2003) No. 4, S. 451-474.

Gebauer, L.; Söllner, M.; Leimeister, J.M. (2012): Hemmnisse bei der Nutzung von Cloud Computing im B2B-Bereich und die Zuordnung dieser zu den verschiedenen Vertrauensbeziehungen. Präsentiert auf der ConLife Academic Conference 2012, Köln, Deutschland. 
Gefen, D.; Karahanna, E.; Straub, D.W. (2003): Trust and TAM in Online Shopping: An Integrated Model. In: MIS Quarterly, Vol. 27 (2003) No. 1, S. 51-90.

Glover, S.; Benbasat, I. (2010): A Comprehensive Model of Perceived Risk of E-Commerce Transactions. In: International Journal of Electronic Commerce, Vol. 15 (2010) No. 2, S. 47-78.

Golkowsky, C.; Vehlow, M. (2011): Cloud Computing im Mittelstand - Erfahrungen, Nutzen und Herausforderungen, PwC, Frankfurt am Main, Germany, 2011.

IDC (2011): Cloud Computing in Deutschland. http://www.idc.de/press/presse_mc cloud2011.jsp, zugegriffen am 28.07.2014.

Jánszky, S.G. (2012): CloudComputing - Der unverstandene Megatrend. www. 2bahead.com/uploads/media/2012-05-11_Trendanalyse_Der_unverstandene Megatrend Cloud.pdf, zugegriffen am 28.07.2014.

Joubert, J.; Belle, J.-P.V. (2009): The Importance of Trust and Risk in M-Commerce: A South African Perspective. Präsentiert auf der 13th Pacific Asia Conference on Information Systems, Hyderabad, Indien.

Kim, D.J.; Ferrin, D.L.; Rao, H.R. (2008): A trust-based consumer decisionmaking model in electronic commerce: The role of trust, perceived risk, and their antecedents. In: Decision Support Systems, Vol. 44 (2008) No. 2, S. 544-564.

Leimeister, J.M.; Ebner, W.; Krcmar, H. (2005): Design, Implementation, and Evaluation of Trust-Supporting Components in Virtual Communities for Patients. In: Journal of Management Information Systems, Vol. 21 (2005) No. 4, S. 101135 .

Luo, X.; Li, H.; Zhang, J.; Shim, J.P. (2010): Examining multi-dimensional trust and multi-faceted risk in initial acceptance of emerging technologies: An empirical study of mobile banking services. In: Decision Support Systems, Vol. 49 (2010) No. 2, S. 222-234.

Marston, S.; Li, Z.; Bandyopadhyay, S.; Zhang, J.; Ghalsasi, A. (2011): Cloud computing - The business perspective. In: Decision Support Systems, Vol. 51 (2011) No. 1, S. 176-189.

Muir, B.M. (1994): Trust in automation: Part I. In: Ergonomics, Vol. 37 (1994) No. 11, S. 1905-1922.

Repschlaeger, J.; Zarnekow, R.; Wind, S.; Turowski, K. (2012): Cloud Requirements Framework: Requirements And Evaluation Criteria To Adopt Cloud Solutions. Präsentiert auf der 20th European Conference on Information Systems (ECIS), Barcelona, Spanien.

Rotter, J.B. (1980): Interpersonal trust, trustworthiness, and gullibility. In: American Psychologist, Vol. 35 (1980) No. 1, S. 1.

Söllner, M.; Leimeister, J.M. (2012): Opening up the Black Box: The Importance of Different Kinds of Trust in Recommender System Usage. Präsentiert auf der 72nd Academy of Management Annual Meeting, Boston, USA. 